\title{
Acceleration of Visual System Development by Environmental Enrichment
}

\author{
Laura Cancedda, ${ }^{1 *}$ Elena Putignano, ${ }^{1 *}$ Alessandro Sale, ${ }^{1 *}$ Alessandro Viegi, ${ }^{1,2}$ Nicoletta Berardi, ${ }^{2,3}$ and \\ Lamberto Maffei ${ }^{1,2}$ \\ ${ }^{1}$ Scuola Normale Superiore, Laboratorio di Neurobiologia, 56100 Pisa, Italy, ${ }^{2}$ Institute of Neuroscience, Consiglio Nazionale delle Ricerche, 56100 Pisa, Italy, \\ and ${ }^{3}$ Dipartimento di Psicologia, Università degli Studi di Firenze, 50100 Firenze, Italy
}

Thus far, the developmental plasticity of the visual system has been studied by altering or reducing visual experience. Here, we investigated whether a complex sensory-motor stimulation, provided by rearing animals in an enriched environment, affects visual system development. We found that raising mice in this condition causes an earlier eye opening, a precocious development of visual acuity, and an accelerated decline of white matter-induced long-term potentiation. These effects are accompanied by a precocious cAMP response element-mediated gene expression and a significant increase of BDNF protein and GAD65/67 expression in enriched pups. In addition, we showed that enriched pups experienced higher levels of licking behavior provided by adult females. Thus, rearing mice from birth in an enriched environment leads to a conspicuous acceleration of visual system development as ascertained at behavioral, electrophysiological, and molecular level.

Key words: environmental enrichment; visual system development; visual evoked potentials; VEP; long-term potentiation; LTP; BDNF; GAD; cAMP/CREB system; maternal care

\section{Introduction}

There is large consensus about the essential role of sensory-driven neural activity in shaping developing circuits in sensory systems. However, this issue has been mostly investigated by paradigms of altered or reduced sensory experience. For instance, classic works on the visual system have used monocular deprivation and dark rearing. The closure of one eye, causing an abnormal unbalanced visual experience, affects the anatomical and functional organization of the visual system. Dark rearing from birth interferes with normal functional maturation of the visual cortex, maintaining neurons in a state of immaturity.

Here, we investigated whether a complex sensory-motor stimulation provided by an enriched environment affects the visual system development. Exposure to enriched environments has been shown to elicit various plastic responses leading to improved learning and memory and to promote structural reorganizations in the brain, especially in the visual cortex (Rosenzweig and Bennett, 1996; Rampon et al., 2000; van Praag et al., 2000; Mohammed et al., 2002). Thus far, most studies on enriched environment were performed using adult animals wherein the

Received May 22, 2003; revised April 6, 2004; accepted April 8, 2004.

This research was supported by Ministero Istruzione Universitá e Ricerca Co Finanziamento 2001 and 2002, Consiglio Nazionale delle Ricerche (CNR) Project SP-5, Progetto Strategico Neuroscienze CNR, Fondo Integrativo Ricerca di Base Projects Ricerca di Base Neuroscienze 01RZH4-002. We thank Dr. T. Pizzorusso for his important assistance in this work. We also thank A. D. Huberman, Dr. G. Ratto, and Prof. A. Fiorentini for critical reading of this manuscript and G. Cappagli and C. Orsini for their technical help.

${ }^{*}$ L.C., E.P., and A.S. contributed equally to this work.

Correspondence should be addressed to Elena Putignano, Laboratorio di Neurobiologia, Scuola Normale Superiore, 56100 Pisa, Italy. E-mail: putignano@in.cnr.it.

D01:10.1523/JNEUROSCI.0845-04.2004

Copyright $\odot 2004$ Society for Neuroscience $\quad$ 0270-6474/04/244840-09\$15.00/0 enriched experience was provided either after weaning or in adulthood, but it is not known whether environmental enrichment can affect the development of the nervous system. Here, we investigated the influence of environmental enrichment on the development of the visual system in animals enriched from birth.

To assess the functional development of the visual cortex, we used the sensitive measure of visual acuity maturation. We found in animals raised in environmentally enriched condition (EC) an acceleration of visual acuity development, assessed both behaviorally by a discrimination task (visual water task) and electrophysiologically by visual evoked potentials (VEPs). Visual system development can be studied in vitro measuring the white matterinduced long-term potentiation (WM-LTP), because its induction is known to undergo an age-dependent developmental decline. Our recordings revealed a precocious developmental decline of WM-LTP in EC with respect to standard condition (SC) reared mice. We surprisingly also observed an accelerated eye opening in EC mice compared with SC mice. In addition, we found that higher levels of licking behavior were provided to enriched pups by adult females.

Recent results have shown that BDNF is increased by enriched rearing condition in adults (Ickes et al., 2000; Pham et al., 2002). BDNF is known to modulate the development and plasticity of the visual cortex (Huang et al., 1999) possibly by regulating the GABAergic inhibitory system, which has a clear role in cortical development (Hensch et al., 1998). We measured BDNF protein and glutamic acid decarboxylase (GAD) 65/67 expression and found that enrichment transiently increases levels of BDNF protein and GAD65/67 expression in the occipital cortex of EC with respect to SC animals before postnatal day (P) 15 .

One of the targets of BDNF signaling is the activation of cAMP 
response element (CRE)-binding protein (CREB), a transcription factor with a well established role in development and plasticity of the visual system either at cortical level (Pham et al., 1999; Cancedda et al., 2003) or, more precociously, at geniculate level (Pham et al., 2001). Enriched environment increases CREB expression and phosphorylation in the hippocampus of adult animals (Williams et al., 2001), but it is not known whether it regulates CRE-mediated gene expression in the developing visual cortex. We found that CRE-mediated gene transcription is developmentally regulated in mouse and that the time course of CRE/ CREB-induced gene expression is robustly accelerated in EC animals, in very good agreement with the precocious functional development of visual cortex and with the precocious increase of BDNF protein level.

\section{Materials and Methods}

Four hundred twenty-one wild-type (WT) C57BL/6J mice and 148 CRELacZ transgenic mice bred with WT C57BL/6J mice were used for the behavioral, electrophysiological, and biochemical experiments. In addition, 59 animals (pups, dams, and "filler" females) have been observed for the assessment of maternal care behavior. The animals were housed in a room with a temperature of $21^{\circ} \mathrm{C}, 12 \mathrm{hr}$ light/dark cycle, and food and water available ad libitum. The eyes of all animals in this study were clear and without obvious optical anomalies.

Timed pregnant female mice were assigned to either environmentally enriched or standard rearing conditions at least $7 \mathrm{~d}$ before delivery. With this procedure, both enriched and standard females received equivalent levels of stress deriving from cage transfer during pregnancy. No difference in gestation time was detected in the two experimental conditions. Also the number of pups in each litter did not significantly differ between EC and SC (6.7 \pm 0.4 and $7.1 \pm 0.4$ pups/litter; Mann-Whitney rank sum test; $p=0.485)$. After birth, all of the litters were housed with their mother until P35.

Rearing environments. Enriched environment consisted of a large cage $(44 \times 62 \times 28 \mathrm{~cm})$ with a wire mesh lid containing several food hoppers, a running wheel, and differently shaped objects (tunnels, shelters, stairs) that were repositioned once per day and completely substituted with others once per week. Every cage housed at least two dams, 10 pups, and two additional filler females. Standard environment consisted of a standard laboratory cage $(26 \times 42 \times 18 \mathrm{~cm})$ housing one litter of pups with their dam or four adults.

Eye-opening observations. From P11, pups ( $n=16$ pups, four litters for $\mathrm{SC} ; n=21$ pups, four litters for EC group) were inspected for eye opening twice per day at $\sim 8: 00$ A.M. and 7:00 P.M. Eye opening was defined as the initial break in the membrane sealing the lids of both eyes.

Assessment of visual acuity. Behavioral assessment of visual acuity started at P19; EC $(n=7)$, untreated SC $(n=10)$, and DMSO- or rolipram-treated SC WT mice $(n=16)$ were trained and tested every $3 \mathrm{~d}$ in the visual water task (Prusky et al., 2000b) to assess their visual acuity. Visual water task trains animals to first distinguish a low $(0.05$ cycles/ degree) spatial frequency vertical grating from gray and then tests the limit of this ability at increasing spatial frequencies. The highest spatial frequency at which $70 \%$ accuracy is achieved is taken as the visual acuity. During each session, the experimenter was blind of the experimental group.

Electrophysiological assessment of visual acuity. A total of 17 SC and 18 EC WT mice was used. Mice were anesthetized with an intraperitoneal injection of $20 \%$ urethane ( $6 \mathrm{ml} / \mathrm{kg}$; Sigma, St. Louis, MO) and mounted in a stereotaxic apparatus allowing full viewing of the visual stimulus. Eyes were not restrained in a fixed position, nor were eyelids kept artificially opened. Body temperature was monitored with a rectal probe and maintained at $37.0^{\circ} \mathrm{C}$ with a heating pad. A large portion of the skull $(4 \times$ $4 \mathrm{~mm}$ ) overlying the binocular visual cortex was drilled and removed leaving the dura intact. A glass micropipette was inserted into the cortex perpendicularly to the stereotaxic plane. In most experiments, microelectrodes were inserted $2.6-3.2 \mathrm{~mm}$ lateral to the $\lambda$ (intersection between sagittal- and lambdoid-sutures) and advanced 100 or $400 \mu \mathrm{m}$ within the cortex. At these depths, VEPs had their maximal amplitude. Electrical signals were amplified (50,000-fold), bandpass filtered (0.3$100 \mathrm{~Hz}$ ), and averaged (at least six events in blocks of 10 events each) in synchrony with the stimulus contrast reversal. Transient VEPs in response to abrupt contrast reversal $(1 \mathrm{~Hz})$ were evaluated in the time domain by measuring the peak-to-baseline amplitude and peak latency of the major component. VEPs in response to a blank stimulus were also frequently recorded to have an estimate of the noise. Visual stimuli were horizontal sinusoidal gratings of different spatial frequency and contrast generated by a VSG2/2 card (Cambridge Research System, Cheshire, UK) and presented on a computer display (mean luminance, $25 \mathrm{~cd} / \mathrm{m}^{2}$; area, $24 \times 26 \mathrm{~cm}$ ) placed $20 \mathrm{~cm}$ in front of the animal. VEP amplitude decreases with increasing stimulus spatial frequency. Visual acuity was determined by linearly extrapolating VEP amplitude to $0 \mathrm{~V}$.

LTP recordings. Brains from 19 SC and 29 EC WT mice were removed and immersed in ice-cold cutting solution containing (in $\mathrm{mM}$ ): $130 \mathrm{NaCl}$, $3.1 \mathrm{KCl}, 1.0 \mathrm{~K}_{2} \mathrm{HPO}_{4}, 4.0 \mathrm{NaHCO}_{3}, 5.0$ dextrose, $2.0 \mathrm{MgCl}_{2}, 1.0 \mathrm{CaCl}_{2}, 10$ HEPES, 1.0 ascorbic acid, 0.5 myo-Inositol, 2.0 pyruvic acid, and 1.0 kynurenate, $\mathrm{pH} 7.3$. Slices $(0.35 \mathrm{~mm}$ thick) of visual cortex were obtained using a Leica (Nussloch, Germany) vibratome. The recording solution was composed as the cutting solution with the following differences (in $\mathrm{mm}$ ): $1.0 \mathrm{MgCl}_{2}, 2.0 \mathrm{CaCl}_{2}, 0.01$ glycine, and no kynurenate. Slices were perfused at a rate of $2 \mathrm{ml} / \mathrm{min}$ with $35^{\circ} \mathrm{C}$ oxygenated recording solution. Electrical stimulation (100 $\mu$ sec duration) was delivered with a bipolar concentric stimulating electrode (FHC, St. Bowdoinham, ME) placed at the border of the white matter and layer VI. Field potentials in layer III were recorded by micropipette (1-3 M $\Omega$ ) filled with $\mathrm{NaCl}(3 \mathrm{M})$. Baseline responses were obtained every $30 \mathrm{sec}$ with a stimulation intensity that yielded a half-maximal response. After achievement of a $10 \mathrm{~min}$ stable baseline (field potential amplitude within $10 \%$ of change and with no evident increasing or decreasing trends), $\theta$ burst stimulation (TBS) was delivered.

BDNF immunoassay. Occipital cortices were dissected (between 8:30 and 9:30 A.M.) from $130 \mathrm{EC}$ and 98 SC mice and were frozen. Both cortices from 8-16 mice were pooled together to represent one sample. Proteins were extracted with lysis buffer (Igepal CA 630 1\%, 10\% glycerol, $20 \mathrm{~mm}$ Tris $\mathrm{HCl}, \mathrm{pH} 8,137 \mathrm{~mm} \mathrm{NaCl}, 0.5 \mathrm{~mm} \mathrm{Na} 3 \mathrm{VO} 4,1 \mu \mathrm{g} / \mathrm{ml}$ Leupeptin, $10 \mu \mathrm{g} / \mathrm{ml}$ Aprotinin, $1 \mathrm{~mm}$ PMSF) and quantified with BioRad (Hercules, CA) protein assay kit. One hundred microliters of BDNF standard and $100 \mu \mathrm{l}$ of samples were run in duplicate (six dilutions) following the protocol from Emax-ImmunoAssay kit (Promega, Madison, WI). Standard samples were run for each plate, and a standard curve was subsequently computed $\left(R^{2}=0.999 \pm 0.001 ; n=6\right)$. Optical absorbance was read at $450 \mathrm{~nm}$ with a microplate reader. Samples were considered BDNF positive when their signal was within the standard curve

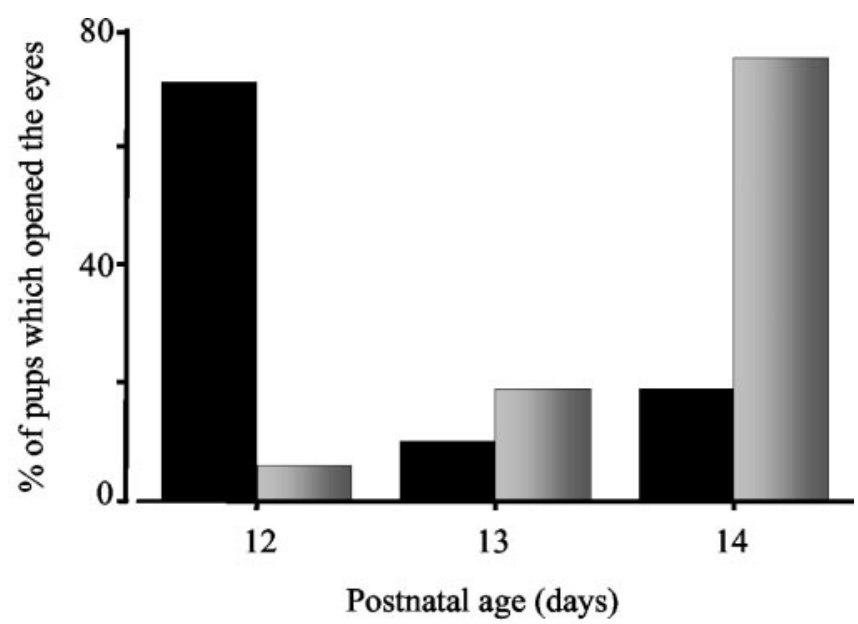

Figure 1. Precocious eye opening in environmentally enriched mice. Percentage of $\mathrm{EC}$ (black) and SC (gray) pups that opened their eyes at the indicated ages is shown. There was a precocious eye opening by $\sim 2 \mathrm{~d}$ (Mann-Whitney rank sum test; $p<0.001$ ) in $\mathrm{EC}$ with respect to SC mice. 
range. To determine the absolute amount of neurotrophin, a titration curve for each sample was generated, and the midpoint of this curve was compared with the standard curve. Data were obtained from two independent experiments, each performed twice.

GAD 65/67 immunoblotting. We used the same samples as for BDNF immunoassay. Ten micrograms of proteins were electrophoresed with $10 \%$ SDS-PAGE and transferred to nitrocellulose. Blots were blocked with $4 \%$ dry milk powder (Bio-Rad), 0.2\% Tween 20 in TBS, and incubated overnight with anti-GAD 65/67 (1 $\mu \mathrm{g} / \mathrm{ml}$ TBS, $2 \%$ milk, $0.1 \%$ Tween 20; Chemicon, Temecula, CA). Blots were incubated with HRP-conjugated secondary antibody (goat anti-rabbit $0.3 \mu \mathrm{g} / \mathrm{ml}$; Bio-Rad), developed by ECL chemiluminescence system (Amersham Biosciences, Arlington Heights, IL).

Maternal care observations. To study maternal care, we used only primiparous females, as typically done in the literature, to avoid the possible confounding effects deriving from the experience of previous motherhood. Four litters were observed for each housing condition. To facilitate behavioral observations in the enriched condition, only one dam with their pups and two additional filler females were housed together. In the SC cages, only one dam was present. In total, the number of animals observed in this study was 59 (43 pups, 8 dams, and 8 filler females). Four EC and four SC cages have been observed. The number of pups per litter was equal between SC and EC dams ( $5.5 \pm$ 0.3 and $5.3 \pm 0.5$ pups/litter, respectively). In both SC and EC groups, maternal behavior was scored daily during six observation sessions of $75 \mathrm{~min}$ each for the first $10 \mathrm{~d}$ postpartum. The observation sessions started at 6:45 A.M., 9:00 A.M., 12:00 P.M., 3:00 P.M., 6:00 P.M., and 9:00 P.M. The first and last sessions were during dark periods in the animal house and were performed under dim red light illumination. For each session, the behavior of each female was scored every $3 \mathrm{~min}$ (150 observations per day). The data have been reported as the percentage of observations in which pups received the target behavior (number of observations in which the target behavior was recorded divided by the total number of observations times 100) (Liu et al., 2000). We focused on licking behavior.

$X$-gal histochemistry. One hundred fortyeight CRE-LacZ transgenic mice (Impey et al., 1996) from both EC and SC groups were used. Genotyping was performed by PCR. CREmediated gene expression was revealed by means of X-gal histochemistry (Impey et al., 1996; Pham et al., 1999; Barth et al., 2000). After the mice were killed (between 8:30 and 9:30 A.M.), brains were dissected out and submerged in ice-cold fixative ( $3 \%$ paraformaldehyde, $0.1 \mathrm{~m} \mathrm{~PB}$ ) for $3 \mathrm{hr}$. Tissue was subjected to two $30 \mathrm{~min}$ washes at room temperature (RT) in solution $\mathrm{A}$ ( $\left.2 \mathrm{~mm} \mathrm{MgCl}_{2}, 10 \mathrm{~mm} \mathrm{PBS}\right)$, one $30 \mathrm{~min}$ wash at RT in solution $\mathrm{B}\left(0.1 \mathrm{M} \mathrm{PB}, 2 \mathrm{~mm} \mathrm{MgCl}_{2}, 0.02 \%\right.$, Igepal CA 630, $0.01 \%$ sodium deoxycholate), and an overnight incubation at $37^{\circ} \mathrm{C}$ in solution $\mathrm{C}$ [solution B plus $5 \mathrm{~mm} \mathrm{~K}_{3} \mathrm{Fe}(\mathrm{CN})_{6}, \mathrm{~K}_{4} \mathrm{Fe}(\mathrm{CN})_{6}$, and $0.6 \mathrm{mg} / \mathrm{ml}$ 5-bromo-4-chloro-3-indolyl- $\beta$-D-galactopyranoside (X-gal; Sigma)]. Tissue was equilibrated in sucrose and frozen and sectioned to $50 \mu \mathrm{m}$ thickness.
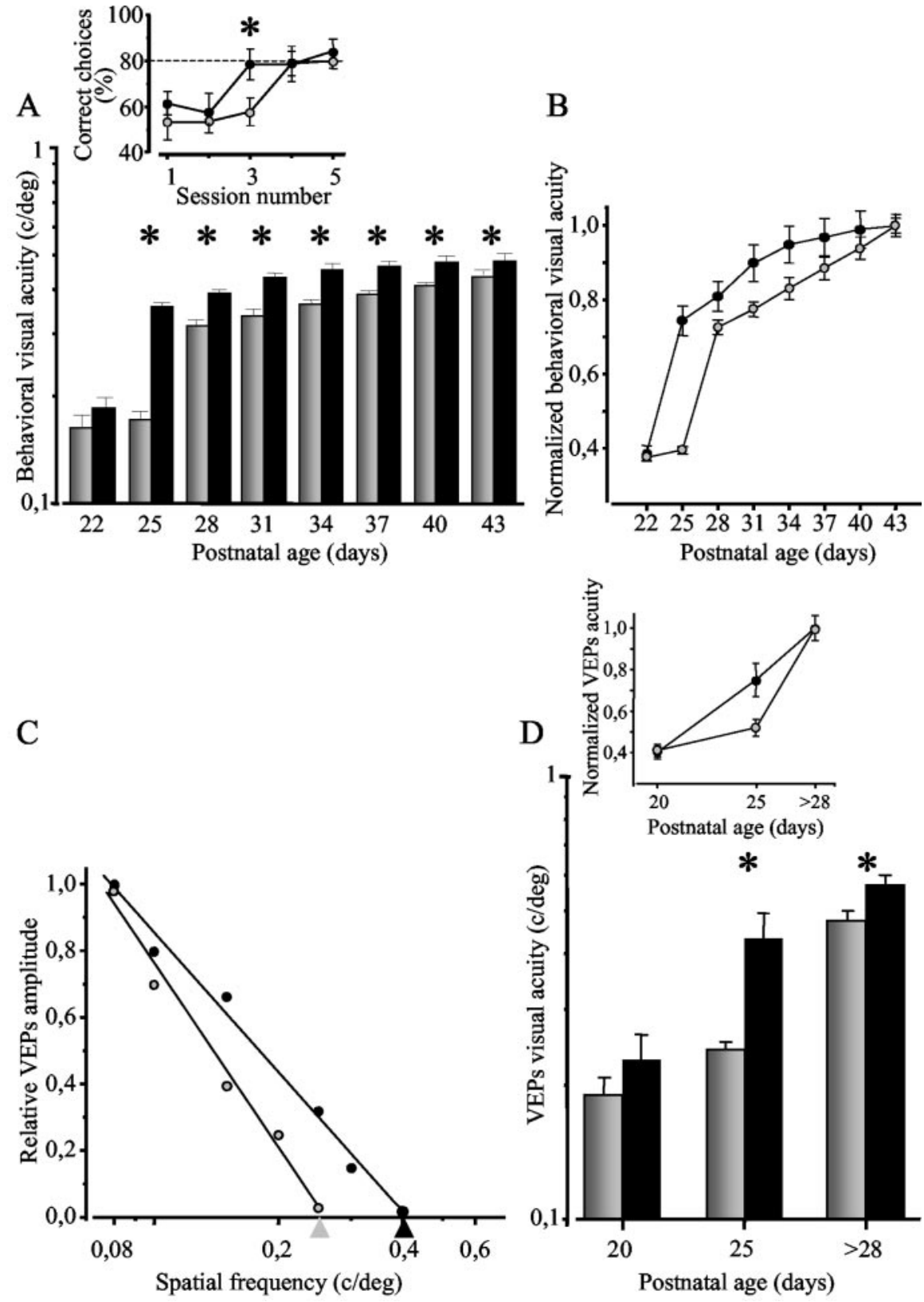

Figure 2. A, Inset, Percentage of correct responses during the training sessions (10 trials in each session) of the visual water task in EC (black) and SC (gray) reared mice. The behavior of the two experimental groups is different at the third session; EC animals have learned the task ( $80 \%$ of correct choices; Kruskal-Wallis one-way ANOVA on ranks; $p<0.001$; post hoc Tukey test; $p<0.05$ ). The bars indicate SEM. The asterisk indicates that the groups are statistically different. $A$, Behavioral assessment of visual acuity in SC (gray) and EC (black) groups at different ages. Visual acuity of animals is plotted as groups of age. Two-way ANOVA shows a significant effect of age and environmental housing condition $(p<0.001)$ and a significant interaction between age and environmental housing condition $(p<0.001)$. Post hoc Tukey test reveals a significant difference ${ }^{*}$ ) at any age starting from P25 between EC and SC groups $(p<0.05)$. The bars indicate SEM. B, Normalized behavioral visual acuity; note that the two developmental curves are clearly shifted along the time axis. C, Examples of visual acuity estimates in an EC and SC mouse at P25. Visual acuity (arrow) was determined by extrapolating VEP amplitude to 0.D, Electrophysiological assessment of visual acuity in SC (gray) and EC (black) mice during postnatal development. Visual acuity of animals is plotted as groups of age. Two-way ANOVA shows a significant effect of age and environmental housing condition $(p<0.001)$ and a significant interaction between age and environmental housing condition $(p<0.05)$. Posthoc Tukey test reveals a significant difference $\left.{ }^{*}\right)$ at any age starting from P25 between $\mathrm{EC}$ and SC groups $(p<0.05)$. The bars indicate SEM. D, Inset, Normalized electrophysiological visual acuity at the indicated ages.

Slices were stained with neutral red to visualize the cortical layers. Blind acquisitions from at least five different slices for each animal were performed.

Visual acuity of SC mice treated with rolipram. Starting at P7 until P28, WT SC animals were given daily saline containing $2 \%$ DMSO $(n=8)$ or 

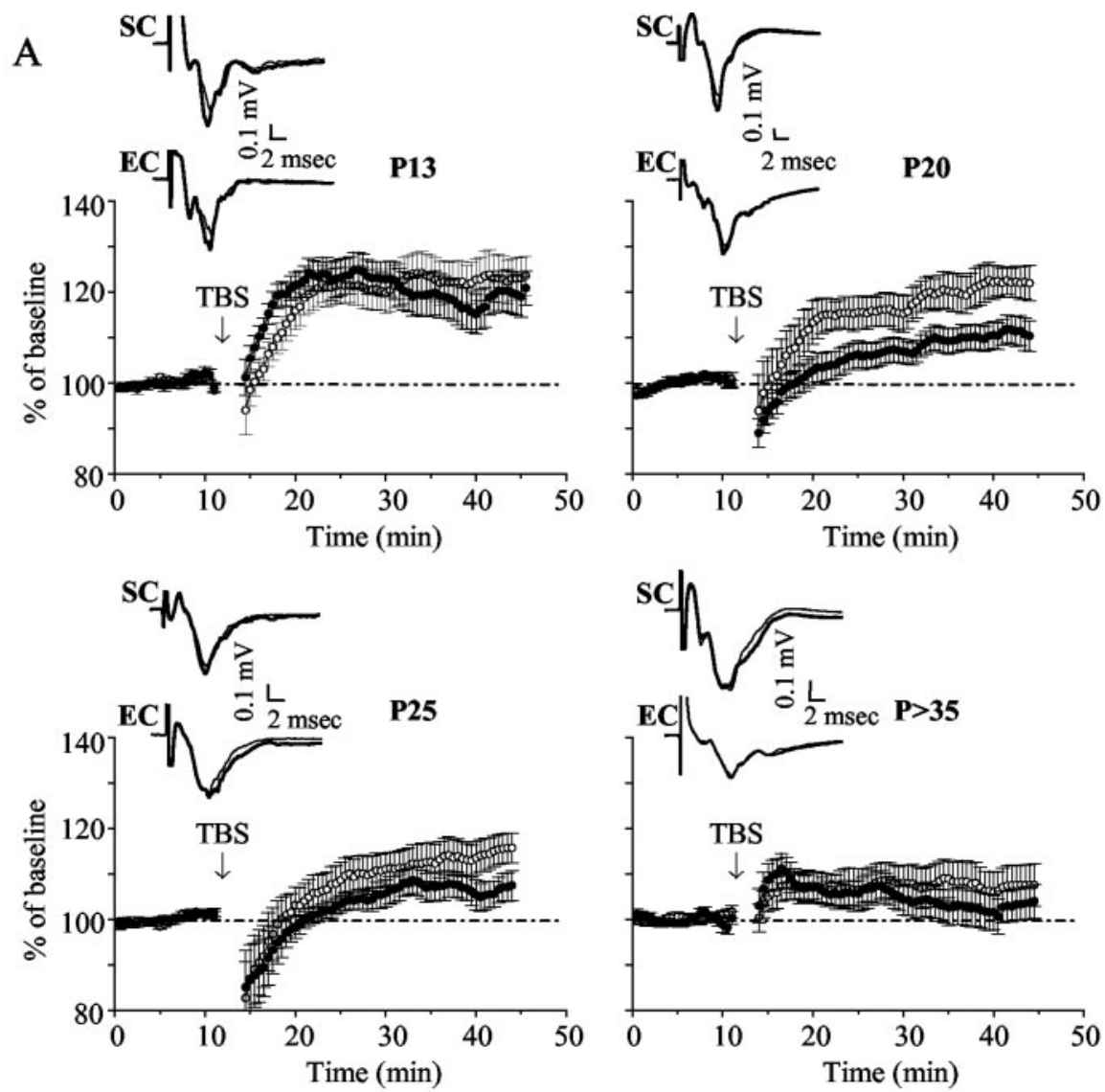

B

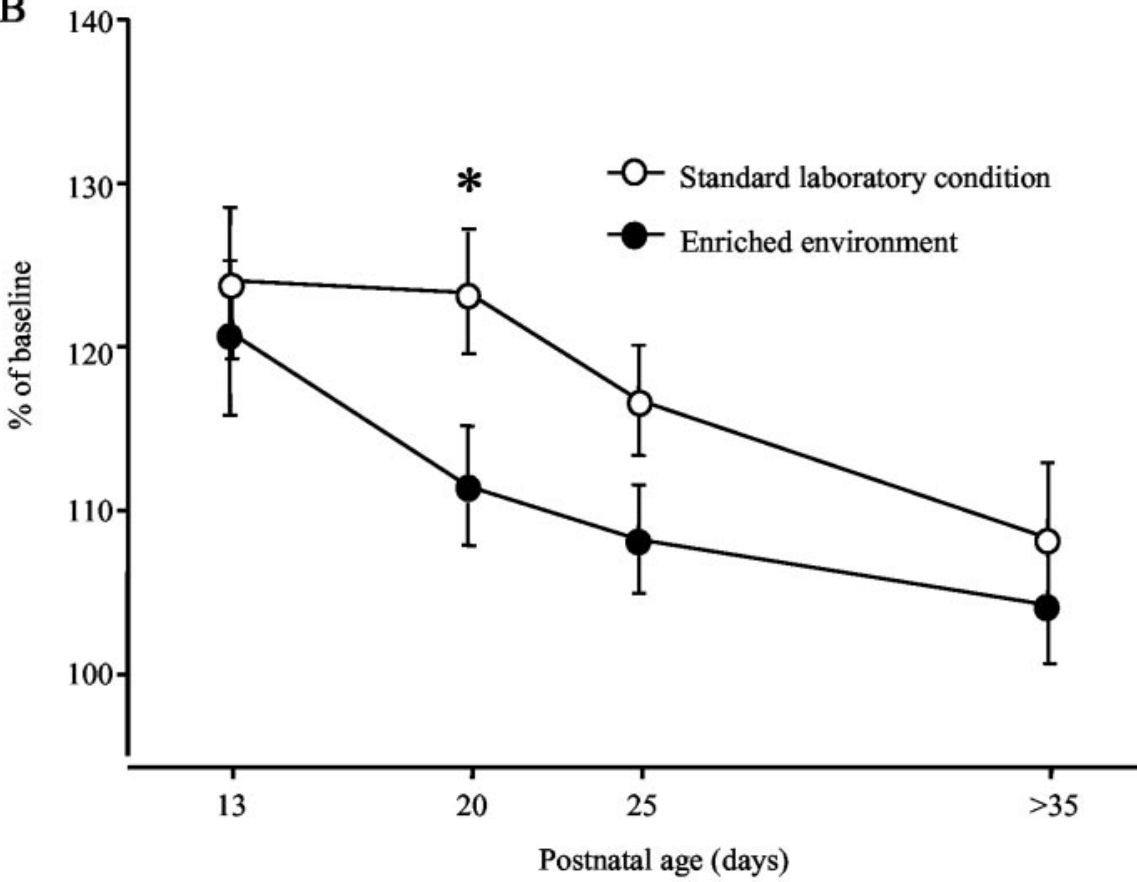

Figure 3. Accelerated developmental decline of WM-LTP in enriched mice. A, Average time course of the changes in layer III field synaptic potential amplitude after TBS of the WM in EC (black) and SC (white) mice at different postnatal ages (P13; $p>35$ ). The bars indicate SEM. Averages of 10 consecutive traces recorded at different postnatal ages from EC and SC mice before (thin line) and $30 \mathrm{~min}$ after (thick line) TBS are also reported on the top of the time course for each age. $B$, Postnatal age-dependent changes in the magnitude ofWM-LTP measured $30 \mathrm{~min}$ after TBS. Three-way ANOVA shows that, although the time course of the field potential amplitude is the same in all conditions $\left(F_{(12)}=0.37 ; p=0.9\right)$, the level of potentiation is significantly different at different ages and for the two different groups $\left(F_{(13)}=2.67, p<0.001\right.$, and $F_{(11)}=29.91, p<0.001$, respectively). Moreover, the three-way ANOVA shows a significant interaction between the postnatal age and the environmental housing $\left(F_{(13,11)}=3.79 ; p=0.01\right)$, with the EC group being significantly different from the SC group at P20 ( posthoc LSD test; $p<0.05$ ). The bars indicate SEM. rolipram $(1.25 \mathrm{mg} / \mathrm{kg}$, i.p., in saline containing $2 \%$ DMSO; $n=8$; Sigma). Animals were trained in the visual water task from $\mathrm{P} 19$ to $\mathrm{P} 21$, and visual acuity was measured at P22, P25, and P28.

\section{Results}

Precocious eye opening in enriched pups

The first physiological event we observed was a precocious eye opening by $\sim 2 \mathrm{~d}$ (Mann-Whitney rank sum test; $p<0.001$ ) in EC with respect to SC mice. The percentage of SC and EC pups that opened their eyes at P12, P13, and P14 was 6 and $71 \%$ at $\mathrm{P} 12,19$ and $10 \%$ at $\mathrm{P} 13$, and 75 and $19 \%$ at P14, respectively (Fig. 1).

\section{Accelerated development of visual acuity in enriched mice}

Visual acuity development was measured in SC and EC mice using a behavioral discrimination task (visual water box) (Prusky et al., 2000a,b). This task challenges the animal's ability to distinguish a grating from homogeneous gray. Mice are first conditioned to distinguish a low spatial frequency grating from the gray, with high reliability (training phase). Each animal's ability limit is then assessed at higher spatial frequencies (visual acuity measurement). SC and EC mice were trained from P19 to P21, and visual acuity was measured once every $3 \mathrm{~d}$ from P22 until P43. Figure $2 A$, inset, shows mean learning curves during the $\mathrm{SC}$ and $\mathrm{EC}$ animal training phase, expressed as the percentage of correct choices per session until animals achieved criterion ( $80 \%$ positive) performance. No difficulty was encountered in training the animals for either the SC and EC groups; nevertheless, EC mice reached $80 \%$ of correct choices criterion by the third session, whereas the SC animals only at the fourth session. As shown in Figure $2 \mathrm{~A}$, SC mice visual acuity was low at P22 $(0.16 \pm 0.01$ cycle/degree $)$ and reached adult levels at P37. At P22, visual acuity of EC mice was similar $(0.19 \pm 0.01$ cycle/ degree) to that of SC mice. In the next few days, however, EC mice visual acuity increased robustly, reaching $0.36 \pm 0.01 \mathrm{cy}$ cle/degree at P25. At this age, visual acuity of EC mice was higher than that of SC mice by more than a factor of 2. EC animal visual acuity reached adult levels at P31 $(0.43 \pm 0.01$ cycle/degree $)$. Thus, in EC mice, visual acuity development was accelerated significantly by $6 \mathrm{~d}$ compared with SC mice. The mature visual acuity of EC and SC mice was also significantly different $(0.49 \pm 0.02$ cycle/degree for EC; $0.44 \pm 0.01$ cycle/degree for SC) but $<20 \%$, as noted by Prusky et al. (2000a).

To avoid possible nonspecific effects resulting from the use of a behavioral task 
in a study involving enriched mice, we measured the visual acuity development also with VEPs. Electrophysiological recordings of VEPs confirmed the behavioral data (Fig. 2C,D). The electrophysiologically measured visual acuity of enriched mice was equal to that of control mice at P20, increased sharply at P25, having a value that was $78 \%$ higher than in controls $(0.24 \pm 0.015$ cycle/degree in control mice; $0.43 \pm 0.06$ cycle/degree in enriched mice; $p<0.001)$, and at P25 it was only $24 \%$ lower than the final value $(0.43 \pm 0.06$ cycle/ degree at P25 vs $0.57 \pm 0.03$ cycle/degree at an age $>$ P28). Standard reared mice increased significantly their visual acuity with respect to the P20 value after P28 (acuity at P25 is not significantly different with respect to $\mathrm{P} 20)$; acuity at $\mathrm{P} 25$ ( $0.24 \pm 0.0156$ cycle/degree) was still $50 \%$ less than the final acuity value $(0.47 \pm 0.03$ cycle/degree $)$. The difference between final acuity in EC and SC mice evaluated electrophysiologically was just $21 \%$, again in line with the behavioral data (Fig. $2 A, D$, inset). Thus, our results cannot be explained simply by a higher final acuity value to be reached in enriched mice but imply an acceleration of the development of visual acuity in enriched subjects.

\section{Precocious developmental decline of WM-LTP in visual cortical slices}

WM-LTP is an in vitro model of visual function development because its induction is known to undergo an age-dependent developmental decline. Indeed, the susceptibility to potentiation of layer III cortical neurons after stimulation of the WM is confined to a critical period in the early postnatal life of rodents and is absent in adult animals (Kirkwood et al., 1995; Huang et al., 1999). We studied the age-dependent decline of LTP in visual cortical slices of EC ( 56 slices) and SC (36 slices) mice. Specifically, we assessed LTP of layer III synaptic responses after TBS of WM at different ages. Figure $3 \mathrm{~A}$ shows the average time course of WM-LTP in SC and EC animals at the indicated ages. The level of potentiation (30 min after TBS) exhibited a different age-dependent decline in EC with respect to SC mice (Fig. 3B). Although at a very early age (P13) substantial WMLTP was found in both experimental groups $(122.8 \pm 4.6 \%$ of preTBS baseline amplitude for SC mice and $119.8 \pm 4.6 \%$ for EC mice), the developmental decline of LTP magnitude in EC animals was faster, with a significant difference between SC and EC groups at P20 (Fig. 3B) (122.1 $\pm 3.6 \%$ for SC mice and $110.6 \pm 3.6 \%$ for EC mice). These results indicate that rearing animals from birth in an enriched environment results in an accelerated decline of WM-LTP.

\section{Higher levels of BDNF protein and GAD65/67 expression in enriched pups}

Because exposure to an enriched environment increases the gene expression of BDNF (Ickes et al., 2000; Pham et al., 2002) in adults, and this neurotrophin modulates, through GABAergic inhibitory system, the plasticity of the visual cortex (Huang et al., 1999), we measured levels of BDNF protein in EC and SC mice. BDNF protein levels were measured using a conventional ELISA protocol. In both SC and EC mice, BDNF protein levels exhibited a developmental increase, which reached a plateau at P20 (Fig. $4 A$, inset). The effect of enrichment was very precocious: EC pups possessed higher levels ( $\sim 55 \%)$ of BDNF at P7 (Fig. 4Apostnatal).

To examine whether the precocious increase in BDNF was paralleled by a change in levels of intra-cortical inhibition, occipital cortices from SC and EC animals at
A

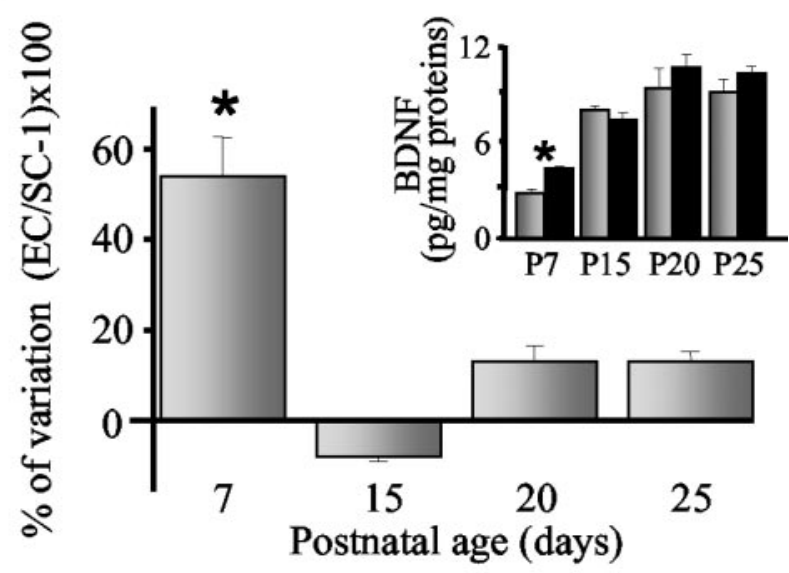

B

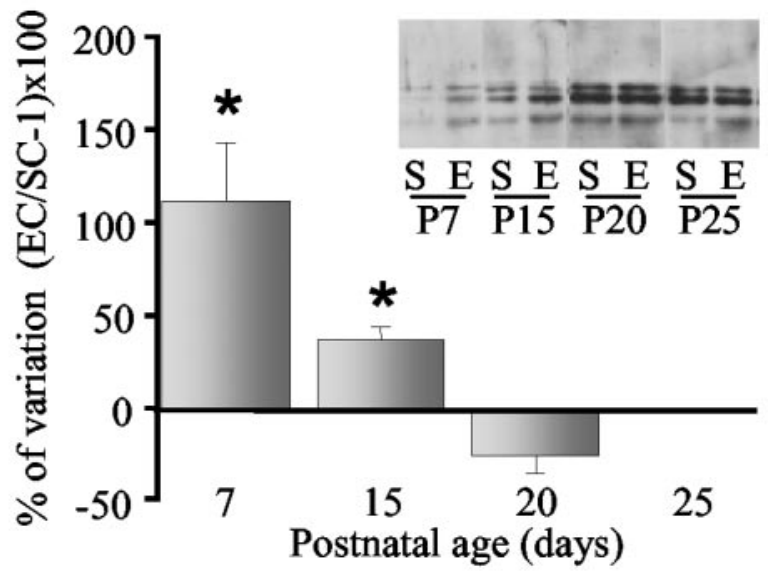

Figure 4. Higher levels of BDNF protein and GAD 65/67 expression in environmentally enriched pups. A, Percentage of variation of BDNF content in EC and SC occipital cortices computed as [(EC/ $S C)-1] \times 100$ at different ages. The asterisk indicates that the BDNF levels in EC and SC groups are different ( $t$ test; $p<0.001)$. Bars indicate SEM. $A$, Inset, Developmental increase of total protein levels of endogenous BDNF in occipital cortices of EC (black) and SC (gray) mice. B, Percentage of variation of GAD 65/67 expression in EC and SC occipital cortices computed as [(EC/SC) - 1] $\times 100$ at different ages. The asteriskindicates that the GAD 65/67 expression in $\mathrm{EC}$ and SC groups is different ( $t$ test; $p<$ 0.001). Bars indicate SEM. $B$, Inset, Representative gel of Western blot for GAD 65/67 expression at different ages in occipital cortices of $\mathrm{SC}$ and $\mathrm{EC}$ mice.

different postnatal ages were analyzed by Western blots for GAD65/67 on the same extract as above. In both SC and EC mice, GAD 65/67 expression exhibited a developmental increase (Fig. $4 B$, inset), but EC pups possessed higher GAD 65/67 levels at P7 $(\sim 112 \%)$ and at P15 ( 37\%) (Fig. $4 B)$.

Figure 5. Involvement of CAMP/CREB system in the development of mice visual cortex. $A$, Precocious massive CRE-mediated gene expression in enriched mice. Brains of CRE-LacZ mice reared in SC and in EC at different ages (P10-P30) are shown. $B$, Quantification of the density of X-gal-positive cells ( $1 \mathrm{~mm}^{2}$ field) for the SC (gray) and EC (black) animals at the indicated ages. Fields were chosen to sample layers II-VI of the binocular visual cortex. The circles represent average density \pm SEM. Two-way ANOVA reveals no significant effect of environmental housing conditions ( $p=0.389)$ but a significant effect of age $(p<0.001)$ and a significant interaction between age and environmental housing conditions $(p<0.001)$. Post hoc Tukey test showed a significant difference at P20 between EC and SC groups $(p<0.05)$. The asterisk indicates that the groups are statistically different. C, Activating the CAMP/CREB system with rolipram partially mimics the enriched environment effects on mice visual acuity development. Behavioral assessment of visual acuity in animals treated with the inhibitor of the phosphodiesterase type IV rolipram (dark gray) and vehicle (light gray) at different ages is shown. Visual acuity of animals is plotted as groups of age. Visual acuity of rolipram-treated animals is significantly different $\left(^{*}\right)$ from vehicle-treated mice at P25 (Kruskal-Wallis one-way ANOVA on ranks, $p<0.001$; post hoc Student-Newman-Keuls test, $p<0.05)$. The bars indicate SEM. 

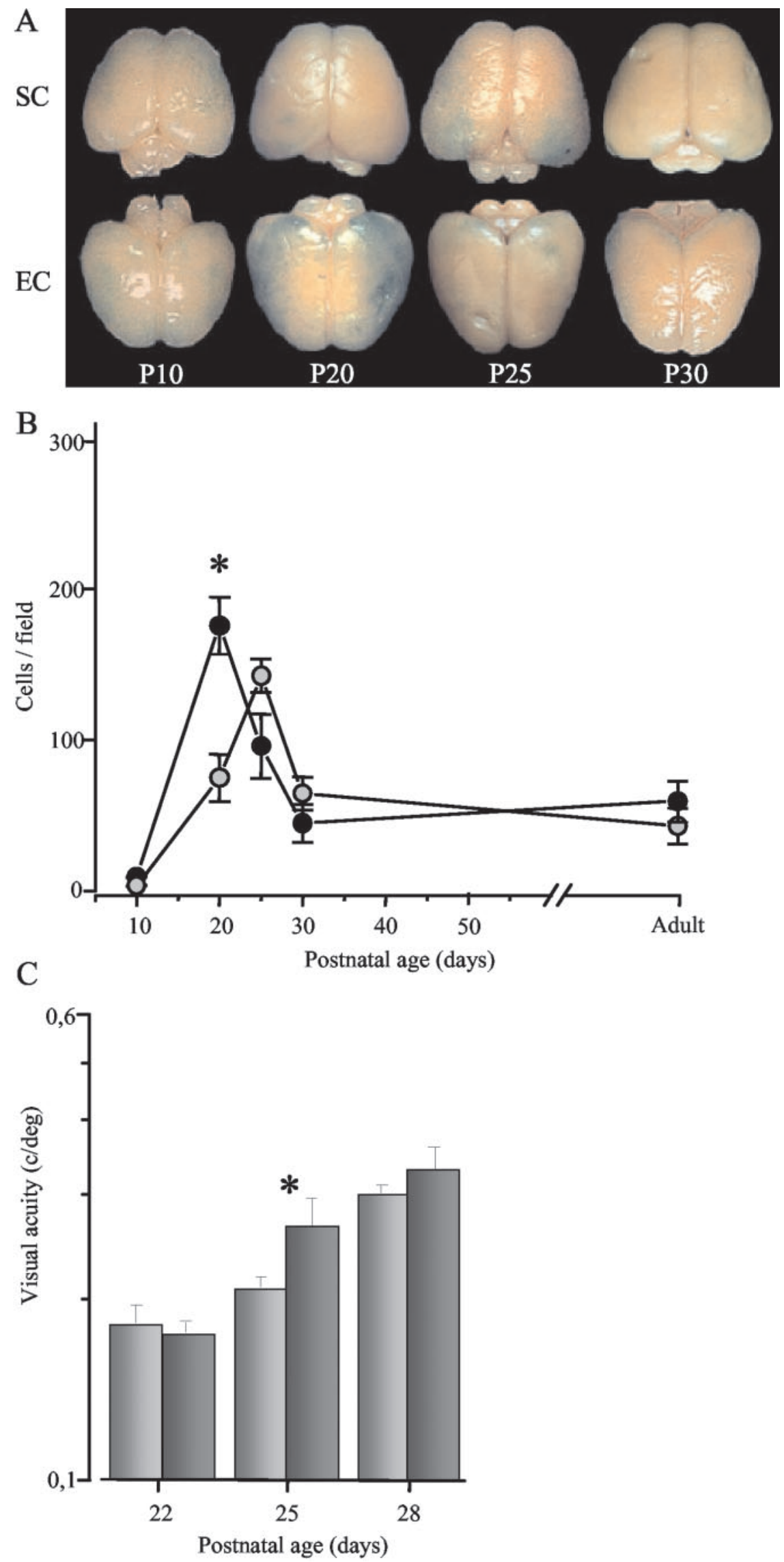

Environmental enrichment and maternal care

The changes in BDNF levels and GABAergic system activation induced by the EC protocol were observed at an early age (P7-P15), when pups still spend most of the time in the nest; preweaning enrichment is likely to interfere with the level of care received by the pups. To assess this, we analyzed maternal behavior in the enriched environment condition and in the standard condition. In particular, we focused on pup licking behavior, because it has been shown that the offspring of mothers that exhibit high levels of pup licking have an increased expression of BDNF in the hippocampus at P8 (Liu et al., 2000) (i.e., about the same age at which we found augmented BDNF levels in the visual cortex of enriched pups). We found that pups reared in the enriched environment received much higher levels of licking than pups reared in standard conditions. Indeed, in measuring the occurrence of licking behavior provided by the mother (and by the other females in the EC cage), we found that the frequency of occurrence of licking (number of observations in which the licking behavior was present over a total of 150 daily observations averaged over the first $10 \mathrm{~d}$ postpartum) received by pups was $13.2 \pm 0.9 \%$ in EC and $5.5 \pm 0.4 \%$ in SC (mean \pm SEM; $n=$ 4 litters per group). The $t$ test revealed a significant effect $(p<0.001)$.

An additional effect exerted on the dams by placing them in an enriched environment could be the change in stress levels caused by changing their housing cage before delivery. However, great care was taken in equaling stressing procedures in standard and enriched dams by transferring both types of dams in a novel cage at least $7 \mathrm{~d}$ before delivery. Furthermore, it has been demonstrated that prenatal stress does not affect eye opening in mice (Meek et al., 2000).

\section{Acceleration of the developmentally regulated CRE-mediated gene expression in EC mice}

An important hub in the development and plasticity of sensory systems and in particular of the visual system is the cAMP/ CREB pathway (Impey et al., 1996; Pham et al., 1999; Barth et al., 2000; Mower et al., 2002; Cancedda et al., 2003). To investigate the involvement of this system in mediating the effects of environmental enrichment on visual system development, we used transgenic mice that carry the $L a c Z$ reporter gene under the control of the CRE promoter (Impey et al., 1996). In 
these animals, the $L a c Z$ gene product, $\beta$-galactosidase, can be visualized as a blue precipitate using X-gal histochemistry, thereby indicating the cells in which CRE-mediated gene expression occurred. Brains from EC and SC animals were processed for $\mathrm{X}$-gal histochemistry at five different ages (Fig. $5 A$ ), and the number of LacZ-positive cells in visual cortical slices was quantified (Fig. 5B). At P10 (before eye opening), brains from both SC and EC mice showed two whitish areas in the occipital cortices (Fig. $5 A$ ). In SC animals, X-gal staining started to increase at P20, peaked at $\mathrm{P} 25$, declined rapidly beginning at $\mathrm{P} 30$, and remained stable into adulthood (Fig. $5 A, B$ ). Notably, in EC mice, gene expression wave occurred precociously, peaking at P20 (Fig. 5B). The EC group showed levels of X-gal staining that were not statistically different from the SC animals at P25, P30, and in adulthood (Fig. $5 B$ ). These data indicate that there is a window of robust CREmediated gene expression in the developing visual cortex and that this event occurs earlier in EC mice.

Because enriched environment is known to affect different brain areas (van Praag et al., 2000), we assessed the effects of our protocol of enrichment on CRE-mediated gene expression also in the somatosensory cortex (S1) in the same mice used to assess it in the visual cortex. Qualitatively, the level of CRE-mediated gene expression was higher in S1 of enriched mice than in standard reared mice at both $\mathrm{P} 10$ and $\mathrm{P} 20$. We performed a quantitative analysis at P10, because $S 1$ develops earlier than visual cortex. We found that the number of lacZ-positive cells in the barrel cortex was $22 \pm 7$ cells $/ \mathrm{mm}^{2}$ in EC mice and $7 \pm 4$ cells $/ \mathrm{mm}^{2}$ in SC mice $(p<0.05)$. Thus, enriched environment influences CREmediated gene expression also in somatosensory cortex, showing that the effect is not specific to the visual cortex. However, the effects in the barrel cortex are evident at a much earlier age (P10) than in the visual cortex (P20), suggesting a correlation with the natural time course of development in the two specific areas.

\section{Activating the cAMP/CREB system with rolipram partially mimics the enriched environment effects on mice visual acuity development}

If the earlier occurrence of the peak in CRE-mediated gene expression wave in the visual cortex is responsible for the effects of enrichment on the functional development of the visual cortex, we should be able to reproduce the effects of enrichment by causing a precocious peak of CREB phosphorylation. This can be accomplished by means of rolipram. This drug is a specific inhibitor of the high-affinity phosphodiesterase type IV and thus activates the cAMP system via inhibition of cAMP breakdown, resulting in an increased phosphorylation of the transcription factor CREB (Tohda et al., 1996; Kato et al., 1998; Nakagawa et al., 2002). CREB is phosphorylated by cAMP-dependent kinase and regulates gene transcription by binding to the CRE on target genes (West et al., 2002). To study whether the cAMP/CREB system is involved in the accelerated maturation of visual acuity in EC mice, we treated SC mice with rolipram. Starting at P7 until P28, SC mice were given saline containing vehicle or rolipram daily. Animals were trained in the visual water task from P19 to P21, and visual acuity was measured at P22, P25, and P28. As shown in Figure $5 C$, the visual acuity of control and rolipramtreated mice was similar at P22 (0.18 \pm 0.01 cycle/degree and $0.17 \pm 0.01$ cycle/degree, respectively). In the next $3 \mathrm{~d}$, the rolipram-treated mice visual acuity significantly increased in comparison with control animals $(0.27 \pm 0.03$ cycle/degree and $0.21 \pm 0.01$ cycle/degree, respectively). At P28, the two groups showed comparable visual acuities $(0.30 \pm 0.01$ cycle/degree for controls; $0.33 \pm 0.03$ cycle/degree for rolipram-treated animals).
These results indicate that CAMP/CREB system activation can modulate visual acuity development in mice, partially mimicking the effect of rearing animals in an enriched environment.

\section{Discussion}

Despite the large interest that enriched environment elicited in the last 50 years, it is not known whether environmental enrichment can affect the development of the nervous system because, so far, most studies were performed in animals that were enriched either after weaning or in adulthood. We demonstrated that rearing mice from birth in an enriched environment leads to a profound acceleration of important properties of visual system development. Even if we focused on the visual system, it is very likely that the complex sensory-motor stimulation provided by the enrichment protocol is not specific to the developing visual system, as suggested by our data on CRE-mediated gene expression in the somatosensory cortex.

The acceleration we found in visual acuity development is by $\sim 6 \mathrm{~d}$, which is a highly significant lapse of time given that the entire critical period for the mouse visual system development lasts only $20 \mathrm{~d}$. This acceleration was evident not only at the behavioral but also at the electrophysiological level and was accompanied by an accelerated decline of WM-LTP. These functional changes were preceded at a molecular level by a significant increase in BDNF protein and GAD 65/67 expression in EC pups, evident at P7, and accompanied by an earlier occurrence of the peak in the wave of CRE-mediated gene expression in the visual cortex.

Enrichment is known to increase BDNF levels in the hippocampus and cerebral cortex in the adult (Falkenberg et al., 1992; Ickes et al., 2000). We found that environmental enrichment also during early postnatal development affects BDNF expression in the visual cortex, where our enrichment protocol from birth induced very precocious changes at P7. Because it is known that BDNF expression strongly affects visual cortex development and plasticity (Huang et al., 1999), the effects of enriched environment that we documented on visual cortical development could be at least partially explained by BDNF precocious expression. However, it is known that enriched environment in the adult increases the expression of other factors that are potentially important for visual system development (neurotrophins, signal transduction molecules, NMDA receptors, GAD 65, CREB, and many others) (Molteni et al., 2002) and, in particular, increases IGF-I expression (Carro et al., 2000; Thoenen and Sendtner, 2002). IGF-I receptors are present in the occipital cortex (Frolich et al., 1998), and IGF-I could therefore influence the expression of molecules relevant for visual cortical plasticity such as nerve growth factor and BDNF itself. We are aware of the complexity of the cellular and molecular changes potentially induced by enriched environment in the developing visual cortex. Additional experiments should be performed to clarify which of these factors is causally involved in the effects of enriched environment on visual cortical development. In the adult, using different models of neurodegeneration, a causal relationship between the protective effects of exposure to enriched environment and the cascade from increased IGF-I to increased BDNF expression, has been demonstrated previously (Carro et al., 2000; Thoenen and Sendtner, 2002); it is not known whether this is true also for the developing brain.

Some of the results that we found in enriched pups, such as BDNF and GAD65/67 protein changes, were observed at a very early age (P7-P15), whereas enrichment in adult mice clearly can influence the subjects directly because they actively interact with 
the environment. The precociousness of the events that we observed in our enriched pups makes it unlikely that they stem from a direct effect from the richness of the environment on them. Rather, the different maternal behavior in enriched conditions with respect to standard conditions could have an important role in the development of the visual system. Indeed, direct behavioral observations of maternal care showed enhanced levels of licking received by enriched pups. The possibility that different levels of maternal care in enriched condition could induce the precocious development of the visual system in our EC animals is in accordance with the results showing that variations in maternal care can affect BDNF levels and neural development of the offspring (Liu et al., 2000). The increased level of licking results from an increased presence of the mother and other females in the nest and provides a strong tactile stimulation, a factor that very likely affects pup development. Indeed, artificial manipulation and tactile stimulation in pups can influence eye opening in rodents (Barnett and Burn, 1967; Smart et al., 1990). In particular, tactile stimulation regulates the expression of hormones important for development (Kuhn and Schanberg, 1998; Schanberg et al., 2003), aids recovery from perinatal cortical lesions, and affects adult pattern of cortical cell dendritic fields (Kolb and Gibb, 1999). Interestingly, the increased levels of tactile stimulation could explain the increased levels of CRE-mediated gene expression that we observed in the somatosensory cortex of EC pups.

We found a clear increase of GAD65 expression at P7 and P15 in EC mice. This strengthens the correlation between BDNF and GABAergic system (Huang et al., 1999). However, it is likely that the inhibitory system is not the only transmitter system affected in the visual cortex by enriched environment. Indeed, enrichment in the adult causes changes in the NMDA receptor expression and in the expression of downstream molecules (Rampon et al., 2000; Molteni et al., 2002). NMDA receptors have been demonstrated to play a crucial role in ocular dominance plasticity and in development of receptive field properties in the visual cortex (Bear et al., 1990; Roberts et al., 1998; Ramoa et al., 2001; Fagiolini et al., 2003) and to be highly sensitive to activity (Quinlan et al., 1999; Philpot et al., 2001). Interestingly, Liu et al. (2000) have shown that maternal care affects NMDAR subunit expression in the hippocampus. If this is also true for the visual cortex, NMDAR could be potentially involved in mediating the effects of increased maternal care on visual cortical development.

Our observation that the peak of the wave of CRE-mediated gene expression in the visual cortex is anticipated in enriched mice suggests that activation of this transcription pathway is a mediator of the effects of enrichment on visual system development. It is indeed known that CREB/CRE participates in the modulation of plasticity and development of the mouse visual cortex and is also developmentally regulated in the LGN (Huang et al., 1999; Pham et al., 1999; Mower et al., 2002). CREB is activated by neuronal activity (West et al., 2002), and environmental enrichment increases immunoreactivity to CREB in the hippocampus (Williams et al., 2001). Therefore, it is conceivable that alterations in the expression-activation of CREB may account for the functional changes between EC and SC animals. Even if, admittedly, we cannot demonstrate a cause-effect relationship between the molecular changes and the behavioral ones, it is interesting to observe that administration of rolipram, an activator of CAMP/CREB system, induced an accelerated development of visual acuity similar to that observed in EC mice. It could be hypothesized that the precocious peak of CRE-mediated gene expression also triggers the other events that we observed in EC with respect to SC mice. It is important to note that the effects on CRE-mediated gene expression could result from the direct effect of the environment on the pups, because at this age, they actively interact with the richness of the environment.

We assessed enriched environment effects on visual cortical development and on behavioral visual acuity, which is the very output of the entire visual system. Therefore, we cannot exclude that enriched environment can influence the development of other stations in the visual pathways. For instance, it could be interesting to assess CRE-mediated gene expression in the LGN, which has a much earlier developmental time course of CREmediated gene expression (Pham et al., 1999), to determine whether enrichment causes an acceleration of this time course. A change in retinal or LGN function could explain some of the effects we observed in the visual cortex. In addition, it will be necessary to assess the development of classical properties such as receptive field size and signal-to-noise ratio in the visual cortex to get a clearer insight on the effects of enrichment on cortical circuits development.

In conclusion, we propose two distinct temporal phases for the effects exerted by enriched environment on visual system development. In the first phase, enhanced levels of maternal care in the enriched condition with respect to the standard condition act as primum movens for molecular changes that could trigger the precocious development of the visual system in our EC animals. Later, after the earlier eye opening $(\sim \mathrm{P} 12)$, when pups begin to actively explore their surroundings, the richness of the environment may directly influence visual system development, for instance, inducing the precocious CRE-mediated gene expression in the visual cortex that indeed was not yet detectable at P10 but only later at P20. This possibility of a direct influence of enriched environment on visual function development is also suggested by a recent study showing that postweaning enrichment leads to normal visual acuity maturation in dark-reared rodents (Bartoletti et al., 2004).

\section{References}

Barnett SA, Burn J (1967) Early stimulation and maternal behaviour. Nature 213:150-152.

Barth AL, McKenna M, Glazewski S, Hill P, Impey S, Storm D, Fox K (2000) Upregulation of cAMP response element-mediated gene expression during experience-dependent plasticity in adult neocortex. J Neurosci 20:4206-4216.

Bartoletti A, Medini P, Berardi N, Maffei L (2004) Environmental enrichment prevents effects of dark-rearing in the rat visual cortex. Nat Neurosci 7:215-216.

Bear MF, Kleinschmidt A, Gu QA, Singer W (1990) Disruption of experiencedependent synaptic modifications in striate cortex by infusion of an NMDA receptor antagonist. J Neurosci 10:909-925.

Cancedda L, Putignano E, Impey S, Maffei L, Ratto GM, Pizzorusso T (2003) Patterned vision causes CRE-mediated gene expression in the visual cortex through PKA and ERK. J Neurosci 23:7012-7020.

Carro E, Nunez A, Busiguina S, Torres-Aleman I (2000) Circulating insulinlike growth factor I mediates effects of exercise on the brain. J Neurosci 20:2926-2933.

Fagiolini M, Katagiri H, Miyamoto H, Mori H, Grant SG, Mishina M, Hensch TK (2003) Separable features of visual cortical plasticity revealed by N-methyl-D-aspartate receptor 2A signaling. Proc Natl Acad Sci USA 100:2854-2859.

Falkenberg T, Mohammed AK, Henriksson B, Persson H, Winblad B, Lindefors N (1992) Increased expression of brain-derived neurotrophic factor mRNA in rat hippocampus is associated with improved spatial memory and enriched environment. Neurosci Lett 138:153-156.

Frolich L, Blum-Degen D, Bernstein HG, Engelsberger S, Humrich J, Laufer S, Muschner D, Thalheimer A, Turk A, Hoyer S, Zochling R, Boissl KW, Jellinger K, Riederer P (1998) Brain insulin and insulin receptors in aging and sporadic Alzheimer's disease. J Neural Transm 105:423-438.

Hensch TK, Fagiolini M, Mataga N, Stryker MP, Baekkeskov S, Kash SF 
(1998) Local GABA circuit control of experience-dependent plasticity in developing visual cortex. Science 282:1504-1508.

Huang ZJ, Kirkwood A, Pizzorusso T, Porciatti V, Morales B, Bear MF, Maffei L, Tonegawa S (1999) BDNF regulates the maturation of inhibition and the critical period of plasticity in mouse visual cortex. Cell 98:739-755.

Ickes BR, Pham TM, Sanders LA, Albeck DS, Mohammed AH, Granholm AC (2000) Long-term environmental enrichment leads to regional increases in neurotrophin levels in rat brain. Exp Neurol 164:45-52.

Impey S, Mark M, Villacres EC, Poser S, Chavkin C, Storm DR (1996) Induction of CRE-mediated gene expression by stimuli that generate longlasting LTP in area CA1 of the hippocampus. Neuron 16:973-982.

Kato H, Araki T, Chen T, Itoyama Y, Kogure K (1998) Effect of rolipram on age-related changes in cyclic AMP-selective phosphodiesterase in the rat brain: an autoradiographic study. Methods Find Exp Clin Pharmacol 20:403-408.

Kirkwood A, Lee HK, Bear MF (1995) Co-regulation of long-term potentiation and experience-dependent synaptic plasticity in visual cortex by age and experience. Nature 375:328-331.

Kolb B, Gibb R (1999) Handbook of developmental cognitive neuroscience (Nelson CA, Luciana M, eds). Cambridge, MA: MIT.

Kuhn CM, Schanberg SM (1998) Responses to maternal separation: mechanisms and mediators. Int J Dev Neurosci 16:261-270.

Liu D, Diorio J, Day JC, Francis DD, Meaney MJ (2000) Maternal care, hippocampal synaptogenesis and cognitive development in rats. Nat Neurosci 3:799-806.

Meek LR, Burda KM, Paster E (2000) Effects of prenatal stress on development in mice: maturation and learning. Physiol Behav 71:543-549.

Mohammed AH, Zhu SW, Darmopil S, Hjerling-Leffler J, Ernfors P, Winblad B, Diamond MC, Eriksson PS, Bogdanovic N (2002) Environmental enrichment and the brain. Prog Brain Res 138:109-133.

Molteni R, Ying Z, Gomez-Pinilla F (2002) Differential effects of acute and chronic exercise on plasticity-related genes in the rat hippocampus revealed by microarray. Eur J Neurosci 16:1107-1116.

Mower AF, Liao DS, Nestler EJ, Neve RL, Ramoa AS (2002) cAMP/Ca ${ }^{2+}$ response element-binding protein function is essential for ocular dominance plasticity. J Neurosci 22:2237-2245.

Nakagawa S, Kim JE, Lee R, Malberg JE, Chen J, Steffen C, Zhang YJ, Nestler EJ, Duman RS (2002) Regulation of neurogenesis in adult mouse hippocampus by cAMP and the cAMP response element-binding protein. J Neurosci 22:3673-3682.

Pham TA, Impey S, Storm DR, Stryker MP (1999) CRE-mediated gene transcription in neocortical neuronal plasticity during the developmental critical period. Neuron 22:63-72.

Pham TA, Rubenstein JL, Silva AJ, Storm DR, Stryker MP (2001) The CRE/ CREB pathway is transiently expressed in thalamic circuit development and contributes to refinement of retinogeniculate axons. Neuron 31:409-420.
Pham TM, Winblad B, Granholm AC, Mohammed AH (2002) Environmental influences on brain neurotrophins in rats. Pharmacol Biochem Behav 73:167-175.

Philpot BD, Sekhar AK, Shouval HZ, Bear MF (2001) Visual experience and deprivation bidirectionally modify the composition and function of NMDA receptors in visual cortex. Neuron 29:157-169.

Prusky GT, Reidel C, Douglas RM (2000a) Environmental enrichment from birth enhances visual acuity but not place learning in mice. Behav Brain Res 114:11-15.

Prusky GT, West PW, Douglas RM (2000b) Behavioral assessment of visual acuity in mice and rats. Vision Res 40:2201-2209.

Quinlan EM, Philpot BD, Huganir RL, Bear MF (1999) Rapid, experiencedependent expression of synaptic NMDA receptors in visual cortex in vivo. Nat Neurosci 2:352-357.

Ramoa AS, Mower AF, Liao D, Jafri SI (2001) Suppression of cortical NMDA receptor function prevents development of orientation selectivity in the primary visual cortex. J Neurosci 21:4299-4309.

Rampon C, Jiang CH, Dong H, Tang YP, Lockhart DJ, Schultz PG, Tsien JZ, Hu Y (2000) Effects of environmental enrichment on gene expression in the brain. Proc Natl Acad Sci USA 97:12880-12884.

Roberts EB, Meredith MA, Ramoa AS (1998) Suppression of NMDA receptor function using antisense DNA block ocular dominance plasticity while preserving visual responses. J Neurophysiol 80:1021-1032.

Rosenzweig MR, Bennett EL (1996) Psychobiology of plasticity: effects of training and experience on brain and behavior. Behav Brain Res 78:57-65.

Schanberg SM, Ingledue VF, Lee JY, Hannun YA, Bartolome JV (2003) PKC alpha mediates maternal touch regulation of growth-related gene expression in infant rats. Neuropsychopharmacology 28:1026-1030.

Smart JL, McMahon AC, Massey RF, Akbar GN, Warren MA (1990) Evidence of non-maternally mediated acceleration of eye-opening in "enriched” artificially reared rat pups. Brain Res Dev Brain Res 56:141-143.

Thoenen H, Sendtner M (2002) Neurotrophins: from enthusiastic expectations through sobering experiences to rational therapeutic approaches. Nat Neurosci 5[Suppl]:1046-1050.

Tohda M, Murayama T, Nogiri S, Nomura Y (1996) Influence of aging on rolipram-sensitive phosphodiesterase activity and $[3 \mathrm{H}]$ rolipram binding in the rat brain. Biol Pharm Bull 19:300-302.

van Praag H, Kempermann G, Gage FH (2000) Neural consequences of environmental enrichment. Nat Rev Neurosci 1:191-198.

West AE, Griffith EC, Greenberg ME (2002) Regulation of transcription factors by neuronal activity. Nat Rev Neurosci 3:921-931.

Williams BM, Luo Y, Ward C, Redd K, Gibson R, Kuczaj SA, McCoy JG (2001) Environmental enrichment: effects on spatial memory and hippocampal CREB immunoreactivity. Physiol Behav 73:649-658. 